\title{
Erratum: Reformulation of the strong-field approximation for light-matter interactions [Phys. Rev. A 93, 023422 (2016)]
}

\author{
A. Galstyan, O. Chuluunbaatar, A. Hamido, Yu. V. Popov, F. Mota-Furtado, P. F. O’Mahony, N. Janssens, \\ F. Catoire, and B. Piraux \\ (Received 8 June 2016; published 3 August 2016)
}

DOI: 10.1103/PhysRevA.94.029901

The authors are grateful to F. H. M. Faisal for drawing their attention to the following correction to the text in the Introduction. In the fourth paragraph, the first line should read, "Later, after the first experimental data on multiphoton ionization was obtained by Voronov and Delone [1,2], Faisal [3] published an approach to above-threshold ionization (ATI) based on the $S$-matrix theory in the velocity gauge. Subsequently, Agostini et al. [4] observed ATI [3], followed by Reiss's [5] version of the $S$-matrix theory also in the velocity gauge."

[1] G. S. Voronov and N. B. Delone, JETP Lett. 1, 42 (1965).

[2] G. S. Voronov, G. A. Delone, N. B. Delone, and O. V. Kudrevatova, JETP Lett. 1, 377 (1965).

[3] F. H. M. Faisal, J. Phys. B 6, L89 (1973).

[4] P. Agostini, F. Fabre, G. Mainfray, G. Petite, and N. K. Rahman, Phys. Rev. Lett. 42, 1127 (1979).

[5] H. R. Reiss, Phys. Rev. A 22, 1786 (1980). 